\title{
Bosonic Resonating-Valence-Bond Description of a Doped Antiferromagnet
}

\author{
Z. Y. Weng, D. N. Sheng, and C. S. Ting \\ Texas Center for Superconductivity and Department of Physics, University of Houston, Houston, Texas 77204-5506
}

(Received 14 October 1997)

\begin{abstract}
We propose a theory for a doped antiferromagnet based on the bosonic resonating-valence-bond (RVB) description incorporating the phase string effect. Both antiferromagnetic and superconducting phase transitions occur naturally inside such a bosonic RVB phase. Two distinct metallic regionsunderdoping and optimum doping - are also found to be a logical consequence; their unique features explain the recent neutron-scattering measurements in cuprates. [S0031-9007(98)06293-0]

PACS numbers: 71.27.+a, 74.20.Mn, 74.72.-h
\end{abstract}

In the study of the $t-J$ model, two distinctive approaches are often used, with the spin degrees of freedom being described either by fermions (slave-boson scheme [1]) or by Schwinger bosons (slave-fermion scheme [2]). Their respective advantage and disadvantage are also well known. In the Schwinger-boson-slave-fermion approach, antiferromagnetic (AF) correlations are usually treated by a bosonic RVB pairing which can produce a variational ground-state energy with unrivaled high precision $[3,4]$ at half filling. But at finite doping this approach is always plagued by the so-called spiral instability [5,6] (spiral twist of spin order) which effectively prevents a controllable study of the metallic phase. In contrast, a metallic phase naturally appears in the fermionic RVB description [1,7] of spins in the slave-boson approach, whereas AF correlations in this kind of metallic state are usually substantially underestimated, especially in a weakly doped regime.

If one adopts the point of view that antiferromagnetism, superconductivity, and anomalous metallic properties all share the same intrinsic origin, the bosonic description of spins may be more appropriate as a starting point since the AF correlations can be properly handled. As for the difficulty of the spiral instability, it may simply reflect the fact that the singular doping effect introduced by holes has been mistreated by mean-field approximations.

Such a singular effect has been recently identified [8] by reexamining the motion of doped holes in the AF background. It has been found that as a hole slowly moves through a closed path it will pick up a nontrivial Berry type phase, which originates from the fact that spin mismatches caused by the hopping of the doped hole cannot be completely "repaired" through spin flips. Such a residual nonrepairable effect is a path-dependent phase product known as phase string [8]. It is a topological effect which would be lost if the phase string effect is averaged out locally at each step of hopping - the reason leading to the aforementioned spiral instability in the mean-field treatment [6] of the slave-fermion scheme. In the onedimensional (1D) case, the same phase string effect is also shown [8] to be responsible for the Luttinger-liquid behavior of the model.
In this Letter, we present an improved two-dimensional (2D) mean-field theory in the spirit of bosonic RVB pairing with incorporating the phase string effect. The spiral instability will be absent in this theory, replaced by the disappearance of the AF long-range order (AFLRO) as holes become mobile (i.e., in metallic phase). Such a new metallic state is drastically different from those in the slaveboson scheme as strong AF correlations remain. The phase string effect plays a crucial role here to put the AFLRO phase, superconducting phase, and normal metallic state within a unified bosonic RVB spin background, which itself is also consistently modified by the phase string effect.

We start with the $t-J$ model, $H_{t-J}=H_{t}+H_{J}$, in the Schwinger-boson, slave-fermion representation [6]

$$
\begin{gathered}
H_{t}=-t \sum_{\langle i j\rangle} \hat{H}_{i j} \hat{B}_{j i}+\text { H.c., } \\
H_{J}=-\frac{J}{2} \sum_{\langle i j\rangle} \hat{\Delta}_{i j}^{s}\left(\hat{\Delta}_{i j}^{s}\right)^{\dagger}
\end{gathered}
$$

where $\hat{H}_{i j}=f_{i}^{\dagger} f_{j}, \quad \hat{B}_{j i}=\sum_{\sigma} \sigma b_{j \sigma}^{\dagger} b_{i \sigma}, \quad$ and $\quad \hat{\Delta}_{i j}^{s}=$ $\sum_{\sigma} b_{i \sigma} b_{j-\sigma}$. Here $f_{i}$ is a fermionic "holon" operator and $b_{i \sigma}$ is known as the Schwinger-boson operator. The bosonic RVB order parameter is defined by [2]

$$
\Delta^{s}=\left\langle\hat{\Delta}_{i j}^{s}\right\rangle \text {. }
$$

At such a mean-field level, in order to gain a finite hopping integral $\left\langle\hat{B}_{j i}\right\rangle \neq 0$, up and down spins have to contribute differently to avoid cancellation due to the sign $\sigma$ appearing in $\hat{B}_{j i}$. But such a mean-field procedure, which leads to a spiral state [6], is fundamentally flawed as the sign $\sigma$ here is the source causing the nonrepairable phase string effect discussed in Ref. [8]. By averaging it out in $\left\langle\hat{B}_{j i}\right\rangle$, the nontrivial topological effect of phase string will be totally lost. To avoid this difficulty, one can introduce a unitary transformation [8] to reformulate the model such that $\hat{B}_{j i}=\sum_{\sigma} e^{i \sigma A_{j i}^{h}} \bar{b}_{j \sigma}^{\dagger} \bar{b}_{i \sigma}$, where the singular sign $\sigma$ is replaced by a link variable $e^{i \sigma A_{j i}^{h}}$. Correspondingly, $\hat{\Delta}_{i j}^{s}$ and $\hat{H}_{i j}$ are redefined by $\hat{\Delta}_{i j}^{s}=\sum_{\sigma} e^{-i \sigma A_{i j}^{h}} \bar{b}_{i \sigma} \bar{b}_{j-\sigma}$ and $\hat{H}_{i j}=e^{i A_{i j}^{f}} h_{i}^{\dagger} h_{j}$, respectively. New spinon and holon operators, $\bar{b}_{i \sigma}$ and $h_{i}$, are both bosonic and such a 
"bosonization" is an interesting consequence of the phase string effect. As justified in Ref. [8], this exact reformulation makes it more suitable for the study of the doped case in both $1 \mathrm{D}$ and $2 \mathrm{D}$ cases. Here the nonlocal gauge field $A_{i j}^{h}$ is defined by a gauge-invariant condition $\sum_{C} A_{i j}^{h}=\pi N_{C}^{h}$ for an oriented closed-path $C$ with $N_{C}^{h}$ being the total hole number enclosed by $C$. And $A_{i j}^{f}$ satisfies $\sum_{C} A_{i j}^{f}=\pi \sum_{\sigma} \sigma \sum_{l \in C} n_{l \sigma}^{b}-\Phi_{C}$ with $n_{l \sigma}^{b}=$ $\bar{b}_{l \sigma}^{\dagger} \bar{b}_{l \sigma}$ and $\Phi_{C}$ referring to a uniform flux enclosed by $C$ with a strength of $\pi$ per plaquette.

Now the phase string effect is precisely tracked through the link variables, $e^{i \sigma A_{i j}^{h}}$ and $e^{i A_{i j}^{f}}$, in $\hat{B}, \hat{\Delta}^{s}$, and $\hat{H}$. We can then consider the mean-field solution characterized by the RVB order parameter defined in Eq. (3). Like the half-filling case [2], a Bogolubov transformation will be used to diagonalize the spin degrees of freedom

$$
\bar{b}_{i \sigma}=\sum_{m}\left(u_{m} \gamma_{m \sigma}-v_{m} \gamma_{m-\sigma}^{\dagger}\right) e^{i \sigma \chi_{m}} \bar{w}_{m \sigma}(i) .
$$

Here $\gamma_{m \sigma}$ is an annihilation operator of spinon excitations and the "single-particle" wave function $\bar{w}_{m \sigma}(i)$ is determined by

$$
\xi_{m} \bar{w}_{m \sigma}(i)=-J_{s} \sum_{j=n n(i)} e^{i \sigma A_{i j}^{h}} \bar{w}_{m \sigma}(j),
$$

with $J_{s}=J \Delta^{s} / 2$. We explicitly introduce a phase factor $e^{i \sigma \chi_{m}}$ in Eq. (4) to show a phase uncertainty in $\bar{w}_{m \sigma}$ which cannot be determined by Eq. (5) as a linear equation. Without changing $\Delta^{s}$, such a "phase" freedom can be fixed by optimizing the hopping integral $\left\langle\hat{B}_{j i}\right\rangle[9]$. The coefficients $u_{m}$ and $v_{m}$ in Eq. (4) are given by $\left(\lambda_{m} / E_{m}+\right.$ $1)^{1 / 2} / \sqrt{2}$ and $\operatorname{sgn}\left(\xi_{m}\right)\left(\lambda_{m} / E_{m}-1\right)^{1 / 2} / \sqrt{2}$, respectively. Here the spinon spectrum is $E_{m}=\sqrt{\lambda_{m}^{2}-\xi_{m}^{2}}$, in which the hopping term only contributes to a shift to the Lagrangian multiplier $\lambda$ by $\lambda_{m}=\lambda-J_{h} / J_{s}\left|\xi_{m}\right|$. The renormalized coupling constant $J_{h}=\langle\hat{H}\rangle t$ will be always chosen as $J_{h}=\delta J$ below ( $\delta$ is the doping concentration). $\lambda$ is determined by the condition $\sum_{i}\left\langle b_{i \sigma}^{\dagger} b_{i \sigma}\right\rangle=$ $N(1-\delta)$, or

$$
2-\delta=\frac{1}{N} \sum_{m} \frac{\lambda_{m}}{E_{m}} \operatorname{coth} \frac{\beta E_{m}}{2}+n_{\mathrm{BC}}^{b},
$$

where $\beta=1 / k_{B} T$ and $n_{\mathrm{BC}}^{b}$ represents the number of spinons per site staying at $E_{m}=0$ state if a Bose condensation $(\mathrm{BC})$ of spinons occurs.

In Fig. 1, the region of a nonzero $\Delta^{s}$ is shown, which practically covers the whole experimentally interested temperature and doping regime. Note that in the bosonic RVB description, $\Delta^{s}$ does not directly correspond to an energy gap, in contrast to the fermionic RVB state [1]. In fact, the spinon spectrum $E_{m}$ is gapless at zero doping and zero temperature [2] which ensures a $\mathrm{BC}$ of spinons. In the new formulation, the transverse spin operator can be written as [8]

$$
S_{i}^{+}=\bar{b}_{i \uparrow}^{\dagger} \bar{b}_{i \downarrow}(-1)^{i} e^{i \Phi_{i}^{h}}
$$

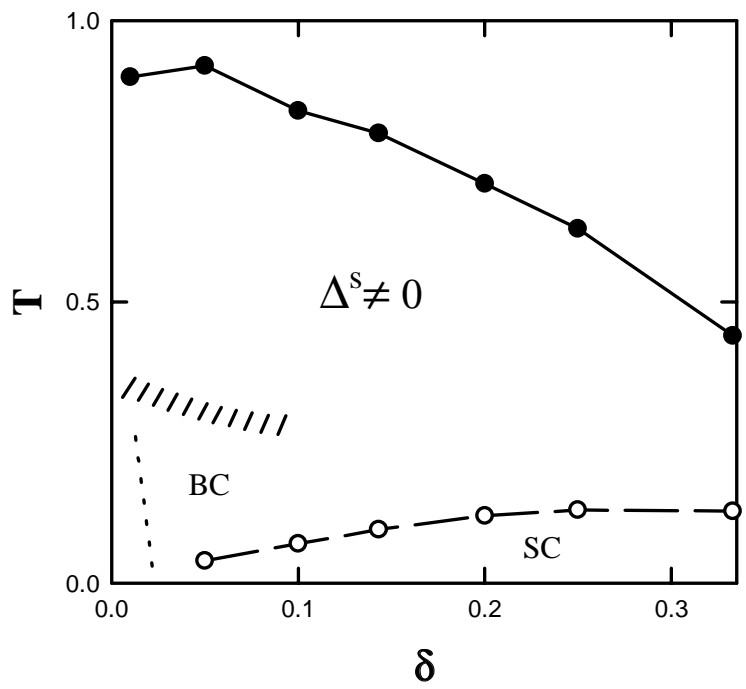

FIG. 1. The phase diagram of a doped antiferromagnet based the bosonic RVB description. The dotted and shaded curves sketch an insulating AFLRO phase and an inhomogeneous metallic region, respectively, described by a spinon Bose condensation (BC). SC indicates the superconducting condensation region determined under an optimal condition (see the text). The temperature $T$ is in units of $J$.

in which

$$
\Phi_{i}^{h}=\sum_{l \neq i} \operatorname{Im} \ln \left(z_{i}-z_{l}\right) n_{l}^{h}
$$

describes vortices (with vorticity $=1$ ) centered on holes $\left(n_{l}^{h}=h_{i}^{\dagger} h_{i}\right)$. In the absence of holes, the BC of spinons will always give rise to $\left\langle S_{i}^{+}\right\rangle \propto(-1)^{i}$, i.e., an AFLRO. But in the presence of mobile holons-in the metallic region-free vortices introduced by $\Phi_{i}^{h}$ will make $\left\langle S_{i}^{+}\right\rangle=0$ even though spinons may be still Bose condensed, resembling a disordered phase in the KosterlitzThouless transition. Only in an insulating phase where holes are localized, the AFLRO may be still sustained as the vortex effect of $\Phi_{i}^{h}$ in Eq. (7) can be "screened" through the compensation of a phase with opposite vorticities generated from spinons (after all, the phase string effect is no longer effective if holes are localized).

So the AFLRO should be absent in the metallic phase, even though the spinon $\mathrm{BC}$ may still persist. In the following, we argue that a spinon $\mathrm{BC}$ phase within the metallic regime must be generally inhomogeneous in charge distribution. Namely, it is a phase separation state. Recall that in the $\mathrm{BC}$ case, $\lambda$ must take a value to make $E_{m}$ gapless such that $n_{\mathrm{BC}}^{b} \neq 0$ can balance the difference between the left and right sides of Eq. (6). Note that the $E_{m}=0$ state corresponds to the maximum of $\left|\xi_{m}\right|$, and thus it is related to those states at the band edge of $\xi_{m}$ which is generally sensitive to the fluctuation of $A_{i j}^{h}$. As $A_{i j}^{h}$ is basically controlled by the holon density, the fluctuations of the charge will then lead to a "Lifshitz" tail in $\xi_{m}$ and play an essential role in determining the $E_{m}=0$ state. Such 
a state is thus associated with inhomogeneous hole configurations so that the Bose condensed spinons form AF orders in hole-deficient region. The detailed nature of the $E_{m}=0$ state will be sensitive to many factors, like the dynamics of holons which is beyond the present approximation. Generally speaking, with the increase of doping, the reduction of the left-hand side of Eq. (6) will eventually make the $\mathrm{BC}$ contribution go away. In Fig. 1 the shaded curve sketches such a BC region which basically defines an underdoped metallic phase. (The dotted curve in Fig. 1 marks the insulating AFLRO phase in the dilute hole regime.)

Superconducting condensation (SC) is characterized by the nonzero average of $\hat{\Delta}_{i j}^{\mathrm{SC}}=\sum_{\sigma} \sigma c_{i \sigma} c_{j-\sigma}$ defined by $[8]$

$$
\hat{\Delta}_{i j}^{\mathrm{SC}}=\hat{\Delta}_{i j}^{s}\left(h_{i}^{\dagger} e^{\frac{i}{2}\left[\Phi_{i}^{s}-\phi_{i}^{0}\right]}\right)\left(h_{j}^{\dagger} e^{\frac{i}{2}\left[\Phi_{j}^{s}-\phi_{j}^{0}\right]}\right)(-1)^{i},
$$

where

$$
\Phi_{i}^{s}=\sum_{l \neq i} \operatorname{Im} \ln \left(z_{i}-z_{l}\right) \sum_{\alpha} \alpha n_{l \alpha}^{b}
$$

describes vortices (antivortices) centered on up (down) spinons, and $\phi_{i}^{0} \rightarrow \phi_{i}^{0} \pm 2 \pi$ after $i$ circles once along a plaquette. Note that the spinons are always paired $\left(\Delta^{s}=\left\langle\hat{\Delta}_{i j}^{s}\right\rangle \neq 0\right)$. In order to have SC, bosonic holons must undergo BC. The vortices described by $\Phi_{i}^{s}$ are all paired up in the ground state whose effect in Eq. (9) is minimal. But at finite temperature, free vortices appear in $\Phi_{i}^{s}$ as spinons are thermally excited from the paired state. In order to achieve the phase coherence in Eq. (9), the condensed holons have to screen those free vortices by forming supercurrents. A phase transition to normal state eventually happens when such screening fails which can be estimated as the free spinon number exceeds the holon number. The $\mathrm{BC}$ of holons will then be interrupted. So the transition temperature $T_{c}$ may be determined by

$$
\left.\frac{2}{N} \sum_{m} \frac{\lambda_{m}}{E_{m}} n\left(E_{m}\right)\right|_{T=T_{c}}=\kappa \delta,
$$

where $\kappa \sim 1$. The left-hand side represents the number of excited spinons determined from $\sum_{i \sigma} b_{i \sigma}^{\dagger} b_{i \sigma}$, with $n\left(E_{m}\right)$ as the Bose function. $T_{c}$ calculated based on Eq. (11) is plotted in Fig. 1 as the dashed curve. It is obtained under the approximation $A_{i j}^{h} \approx \bar{A}_{i j}^{h}$ in Eq. (5) $\left(\bar{A}_{i j}^{h}\right.$ is defined by $\left.\sum_{C} \bar{A}_{i j}^{h}=\pi\left\langle N_{c}^{h}\right\rangle\right)$. The value of $T_{c}$ in Eq. (11) depends on a characteristic low-energy scale of spinons to be identified later in the dynamic susceptibility function. Finally, the symmetry of the SC order parameter may be determined as

$$
\left\langle\hat{\Delta}_{i i+\hat{x}}^{s}\right\rangle /\left\langle\hat{\Delta}_{i i+\hat{y}}^{s}\right\rangle=e^{-i \frac{1}{2} \sum_{\square} \Delta \phi_{j k}^{0}}=-1,
$$

where $\sum_{\square}$ denotes a summation of $\Delta \phi_{j k}^{0} \equiv \phi_{j}^{0}-\phi_{k}^{0}$ over four links of a plaquette and the result indicates a $d$-wave symmetry for the nearest-neighboring SC pairing.

Thus, in the metallic phase there exist two temperature scales: $T_{\mathrm{BC}}$ and $T_{c}$. At low doping where $T_{\mathrm{BC}}>T_{c}$, the charge inhomogeneity or phase separation happens below $T_{\mathrm{BC}}$ and further below $T_{c}$ holons are also expected to be condensed into nonuniform regions in favor of the spin correlation energy. On the contrary, once $T_{c}>$ $T_{\mathrm{BC}}$, holons will experience $\mathrm{BC}$ first and be uniformly distributed in real space. For the ideal BC of holons, $A_{i j}^{h}$ can be replaced by $\bar{A}_{i j}^{h}$ with a substantial reduction of $\delta A_{i j}^{h}=A_{i j}^{h}-\bar{A}_{i j}^{h}$ below $T_{c}$. The spinon spectrum is then qualitatively changed below $T_{c}$ which prevents spinons from Bose condensing into an inhomogeneous phase at lower temperature (see below). $T_{c}$ shown in Fig. 1 is estimated under such a condition and it optimizes $T_{c}$ as compared to the case with stronger fluctuations in $A_{i j}^{h}$. This regime may be properly defined as the optimumdoping regime in our theory. Beyond $T_{c}>T_{\mathrm{BC}}$, a crossover due to statistics transmutation may quickly set in as holons tend to be always Bose condensed even at high temperature such that spinons have to be turned into fermions, which leads to the breakdown of the bosonic RVB state and is beyond the scope of the present paper.

How can two regions of the metallic phase be distinguished by experiment? Here we would like to focus on a direct experimental signature in the local dynamic spin susceptibility $\chi_{L}^{\prime \prime}(\omega) . \quad \chi_{L}^{\prime \prime}(\omega)$ is given by

$$
\begin{gathered}
\chi_{L}^{\prime \prime}(\omega)=\frac{\pi}{4} \sum_{m m^{\prime}} K_{m m^{\prime}}\left\{1 / 2\left[1+n\left(E_{m}\right)+n\left(E_{m}^{\prime}\right)\right]\left(u_{m}^{2} v_{m^{\prime}}^{2}+v_{m}^{2} u_{m^{\prime}}^{2}\right) \delta\left(|\omega|-E_{m}-E_{m^{\prime}}\right)+\left[n\left(E_{m}\right)-n\left(E_{m^{\prime}}\right)\right]\right. \\
\left.\times\left(u_{m}^{2} u_{m^{\prime}}^{2}+v_{m}^{2} v_{m^{\prime}}^{2}\right) \delta\left(\omega+E_{m}-E_{m^{\prime}}\right)\right\}
\end{gathered}
$$

where $\omega>0$ with $K_{m m^{\prime}} \equiv \sum_{i \sigma}\left|w_{m \sigma}(i)\right|^{2}\left|w_{m^{\prime} \sigma}(i)\right|^{2}$. As shown in Fig. 2, $\chi_{L}^{\prime \prime}(\omega)$ exhibits a double-peak structure in low-energy region for the spinon $\mathrm{BC}$ case. The lowest peak in Fig. 2 originates from the excitations of the Bosecondensed spinons which disappears above $T_{\mathrm{BC}}$, while the second peak is contributed by regular spinon pairs excited from the vacuum. Here $\delta A_{i j}^{h}$ is treated as a random flux, with a strength of the flux per plaquette $\delta \phi=0.3 \pi \delta$ at $\delta=1 / 7 \approx 0.143$. Corresponding $T_{\mathrm{BC}}$ is found to be $\sim 0.21 \mathrm{~J}$. On the other hand, if the holon BC happens first with $A_{i j}^{h} \approx \bar{A}_{i j}^{h}(\delta \phi=0)$ below $T_{c}$, then the spinon BC is found absent and only a single peak is left, as shown in the inset of Fig. 2. Here the sharpness of the peak is due to the Landau-level effect caused by $\bar{A}_{i j}^{h}$ in $E_{m}$. Its corresponding energy scale also determines $T_{c}$ in Fig. 1 according to Eq. (11). Such a sharp peak structure will be qualitatively changed above $T_{c}$ as no more Bose condensed holons exist and the fluctuation part of $A_{i j}^{h}$ becomes dominant.

Neutron-scattering measurement has indeed revealed a double-peak structure in $\mathrm{YBa}_{2} \mathrm{Cu}_{3} \mathrm{O}_{6.5}$ compound recently 


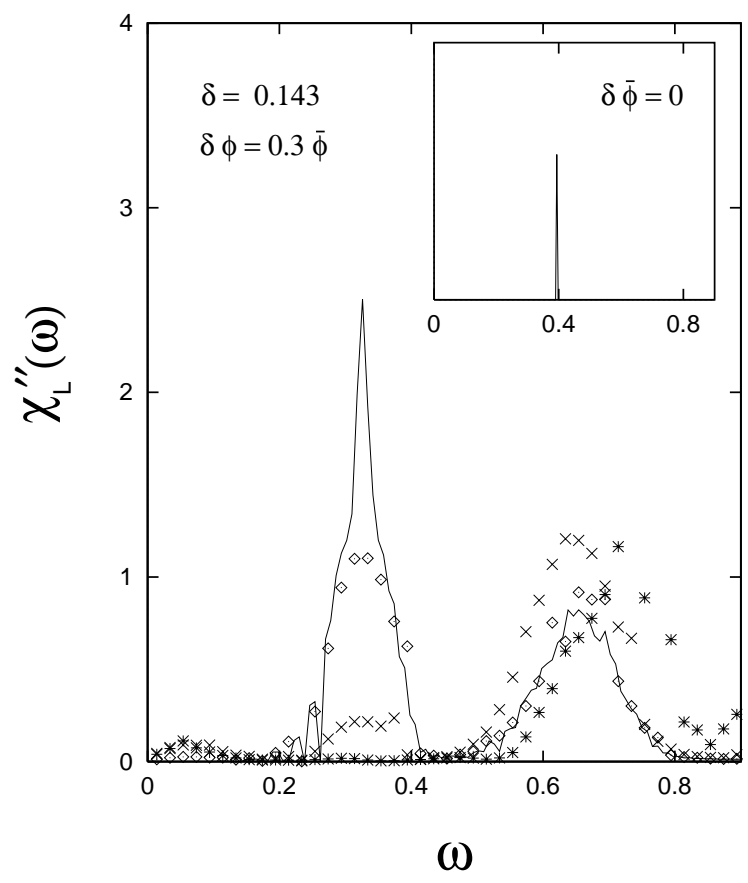

FIG. 2. Local dynamic spin susceptibility $\chi_{L}^{\prime \prime}(\omega)$ vs $\omega$ (in units of $J$ ) at $\delta=0.143$. Solid curve: $T=0 ;(\diamond): T=0.1$; $(\times): T=0.2 ;(*): T=0.3$. Here $T_{\mathrm{BC}}=0.21$ with $\delta \phi=$ $0.3 \phi^{-}$. The inset: $\chi_{L}^{\prime \prime}(\omega)$ vs $\omega$ under the optimal condition: $\delta \phi=0$ at $T=0$.

[10], where the lower peak is located near $30 \mathrm{meV}$ and the second one is, around $60 \mathrm{meV}$, about twice bigger in energy as predicted by the theory (Fig. 2). Thus, this underdoped material can be understood in the present theory as in the spinon BC phase. On the other hand, a "resonancelike" sharp peak at $41 \mathrm{meV}$ has been well known for $\mathrm{YBa}_{2} \mathrm{Cu}_{3} \mathrm{O}_{7}$ below $T_{c}$ [11], which is consistent with the case shown in the inset of Fig. 2 if $J \sim 100 \mathrm{meV}$. Namely, it corresponds to the uniform phase without the spinon BC, defined as an optimum metallic region in the theory with an optimized $T_{c}$. Magnetic peaks located near $\mathbf{Q}_{0}=(\pi, \pi)$ are also identified in the momentum space at the "resonance" energy in both the experiment and theory, suggesting an AF nature of spin fluctuations.

Therefore, by incorporating the singular doping effect-phase string, we are able to generalize the bosonic RVB description from half-filling case to the metallic regime. While the bosonic RVB pairing, representing short-range AF correlations, is the driving force behind the antiferromagnetism and superconductivity, it is the combination with the phase string effect that decides when and where they occur in the phase diagram. Two kinds of metallic state-underdoping (with phase separation) and optimum doping (uniform phase) are also predicted by the present theory with distinct experimental features. As the key structure in this theory, the spin and charge excitation spectra are mutually influenced through the topological gauge field $A_{i j}^{h}$ and $A_{i j}^{f}$ as the manifestation of the phase string effect. The example in the optimal-doping case shows how it works: a uniformly distributed Bosecondensed holons leads to a sharp peak in the spinon spectrum through $A_{i j}^{h}$ below $T_{c}$. And the gap in the spinon excitations in turn sustains such a SC state until a sufficient amount of excited spinons destroys the holon $\mathrm{BC}$ via $A_{i j}^{f}$ at higher temperature. In the normal state, the sharp peak in the spin excitation spectrum will be gone due to the drastically modified $A_{i j}^{h}$, while the scattering of holons with $A_{i j}^{f}$ will dictate the transport properties. The spinon $\mathrm{BC}$ and charge inhomogeneity in the underdoped case are similarly connected through the same gauge fields. The spinon BC also bears some resemblance to a "pseudogap" phenomenon in the behavior of uniform spin susceptibility, resistivity, etc., which will be discussed elsewhere.

The present work is supported by grants from the Texas ARP and the Robert A. Welch foundation, and by Texas Center for Superconductivity at University of Houston.

[1] P. W. Anderson, Science 235, 1196 (1987); Z. Zou and P. W. Anderson, Phys. Rev. B 37, 627 (1988).

[2] D. P. Arovas and A. Auerbach, Phys. Rev. B 38, 316 (1988).

[3] S. Liang, B. Doucot, and P. W. Anderson, Phys. Rev. Lett. 61, 365 (1988).

[4] Y.-C. Chen and K. Xiu, Phys. Lett. B 181, 373 (1993); Y.-C. Chen, Mod. Phys. Lett. B 8, 1253 (1994).

[5] B. I. Shraiman and E. D. Siggia, Phys. Rev. Lett. 62, 1564 (1989); 61, 467 (1988).

[6] C. Jayaprakash et al., Phys. Rev. B 40, 2610 (1989); D. Yoshioka, J. Phys. Soc. Jpn. 58, 1516 (1989); C. L. Kane et al., Phys. Rev. B 41, 2653 (1990); Z. Y. Weng, Phys. Rev. Lett. 66, 2156 (1991).

[7] See X. G. Wen and P. A. Lee, Phys. Rev. Lett. 76, 503 (1996), and the references therein.

[8] D. N. Sheng, Y. C. Chen, and Z. Y. Weng, Phys. Rev. Lett. 77, 5102 (1996); Z. Y. Weng, D. N. Sheng, Y. C. Chen, and C. S. Ting, Phys. Rev. B 55, 3894 (1997).

[9] Z. Y. Weng, D. N. Sheng, and C. S. Ting (unpublished).

[10] P. Bourges, et al., Phys. Rev. B 56, R11 439 (1997).

[11] M. A. Mook et al., Phys. Rev. Lett. 70, 3490 (1990); H. F. Fong et al., Phys. Rev. Lett. 75, 316 (1995). 